\title{
Does Exercise Have a Preventive Effect on Secondary Lymphedema in Breast Cancer Patients Following Local Treatment? - A Systematic Review
}

\author{
Freerk T. Baumann $^{a} \quad$ Alexandra Reike ${ }^{a}$ Michael Hallek $^{a} \quad$ Joachim Wiskemann $^{b}$ \\ Vanessa Reimer ${ }^{\mathrm{a}}$ \\ a Department I of Internal Medicine, Center of Integrated Oncology Cologne Bonn, University Hospital of Cologne, Cologne, Germany; \\ ${ }^{b}$ Department of Medical Oncology, National Center for Tumor Diseases (NCT), Heidelberg University Hospital, Heidelberg, Germany
}

\section{Keywords}

Breast cancer - Lymphedema - Exercise - Prevention . Prophylaxis

\section{Summary}

Background: Secondary lymphedema (SL) is a possible side effect of breast cancer treatment. Current data describe a positive influence of exercise on upper lymphedema. This systematic review evaluates studies examining a potential preventive effect of exercise on $\mathrm{SL}$ incidence. Methods: A systematic literature search of PubMed, LIVIVO, and the Cochrane Library was performed. Results: 8 randomized controlled trials were included in the review. 3 studies investigated the effect of strength training, and 5 the effect of combined exercise therapy. 5 studies included participants without lymphedema at study entry, and 3 studies included both patients with and without lymphedema. The partly significant results showed that progressive strength training as well as combined dynamic exercise therapies consisting of physiotherapy, physical therapy, and/or kinesiotherapy are safe and can prevent SL. Onset as well as diagnosis of lymphedema were significantly decreased in 5 studies in the exercise group. Conclusion: All 8 studies included indicate a potential preventive effect of exercise on SL; however, further research is needed.

(C) 2018 S. Karger GmbH, Freiburg

\section{Introduction}

Over 1.8 million women worldwide are diagnosed with breast cancer every year [1]. Due to improved medical treatment options and early screening and detection, breast cancer survival rates have increased significantly in the last years, and more than $80 \%$ of patients can be cured [1]. Given the improved survival rates, dealing with treatment-associated side effects such as secondary lymphedema (SL) is a new challenge in cancer care [2]. SL is likely to occur after the surgical removal of lymph nodes or in conjunction with radiotherapy [3]. SL is generally described as 'arm swelling and dysfunction' [4] and is defined as an increase in arm circumference by more than $2 \mathrm{~cm}$ [5] or as an accumulation of excessive protein-rich liquid in a part of the body where lymphatic vessels have been damaged [6]. About $20-30 \%$ of all breast cancer patients develop SL [7].

The American College of Sports Medicine (ACSM) roundtable on exercise guidelines for cancer survivors describes that exercise during and after cancer treatment is safe and can help patients improve their physical capacity and quality of life [8]. The ACSM guidelines indicate specific exercise programs oriented towards impairments associated with disease and medical treatment [8]. Strength exercise does not have any negative effects on an existing SL [9]; instead, it has beneficial effects such as improvement of strength [2] and lower exacerbation rates [10].

However, these recommendations do not include any information about the prevention of SL in breast cancer patients. According to our knowledge, no systematic review on solely the topic of prevention has been published so far. Therefore, we conducted a systematic review to analyze possible preventive effects of exercise on the incidence of SL in breast cancer patients.

\section{KARGER}

(๑) 2018 S. Karger GmbH, Freiburg
PD Dr. Freerk T. Baumann 
Table 1. Inclusion and exclusion criteria
Inclusion criteria

- Randomized controlled trials

- Female sex

- Studies including only subjects without secondary lymphedema at study entry

- Mixed studies including subjects with and without secondary lymphedema at study entry

- Surgical removal of at least 1 lymph node

- Exercise including manual lymphatic drainage

- Studies were included from 2006 to 2016 (June)

- Studies including at least 20 subjects

- Studies in English and German language

Exclusion criteria

- Male sex

- Studies including only subjects with secondary lymphedema at study entry

- No surgical removal of any lymph nodes

- Reviews

- Case reports

- Expert opinions

- Animal studies

- Studies combining exercise and nutrition

\section{Methods}

In order to analyze the possible preventive effect of exercise on SL in breast cancer patients, a comprehensive literature search was performed. 3 reviewers independently searched the available literature in the PubMed, LIVIVO, and Cochrane Library databases in order to identify randomized controlled exercise intervention studies with breast cancer patients focusing on the prevention of SL. The literature search was completed in June 2016. The keywords 'breast cancer', 'lymphedema', 'prevention', 'exercise', 'physical activity', 'physical fitness', 'physical exercise', 'sport endurance', 'resistance training', 'strength training', 'weight training', 'physiotherapy', 'physical therapy', 'kinesiotherapy', 'movement, 'aerobic', and 'sport' were used. Only randomized and controlled studies with more than 20 subjects were included. Parameters such as inclusion and exclusion criteria were defined and are listed in table 1.

\section{Results}

A total of 8 randomized controlled intervention studies investigating the preventive effects of exercise on the development of SL in breast cancer patients were identified (tables 2, 3, fig. 1). The studies by Schmitz et al. [11], Sagen et al. [12], Torres Lacomba et al. [5], De Rezende et al. [13], and Zimmermann et al. [14] enrolled only subjects without lymphedema at study entry. The studies by Ahmed et al. [15], Devoogdt et al. [16], and Zhang et al. [17] included both subjects with and without lymphedema at study entry. In total, 1,810 participants were investigated, and 1,780 of these were included in the post-analysis. 5 studies provided details on age; participants were on average 54 years old. 4 studies reported that their subjects received radiotherapy, chemotherapy, or hormone therapy during the intervention period.

The exercise intervention comprised either resistance training $(n=3)[11,12,15]$ or a combined exercise therapy $(n=5)$ consisting of physiotherapy, physical therapy, manual lymphatic drainage

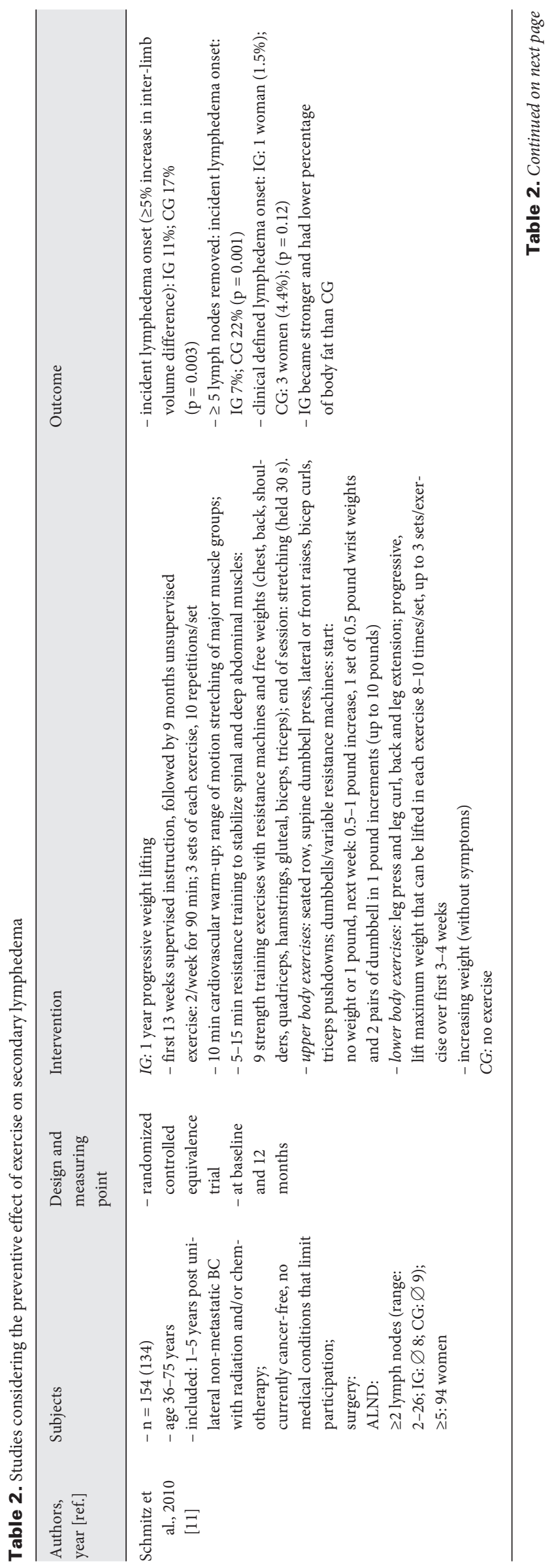




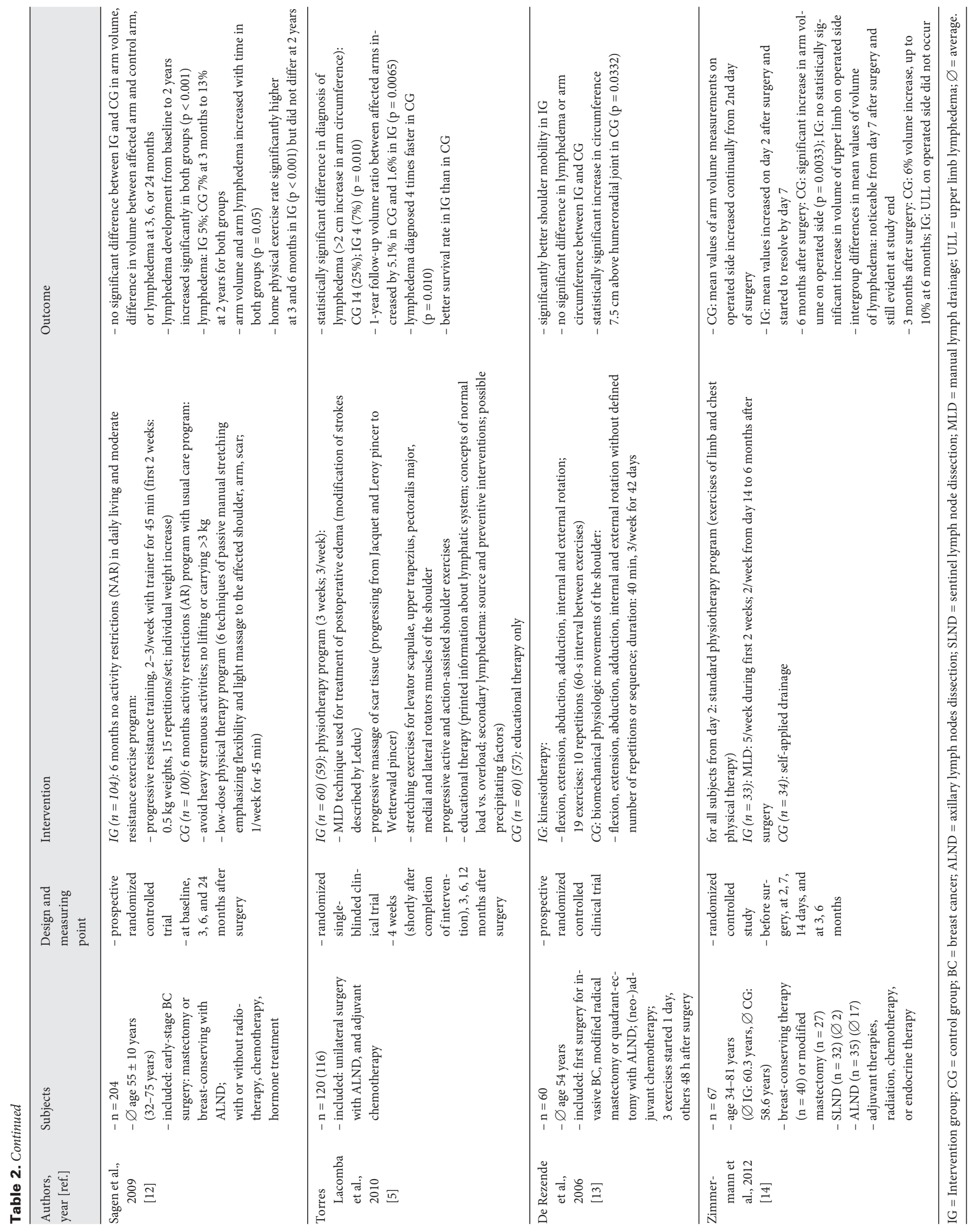




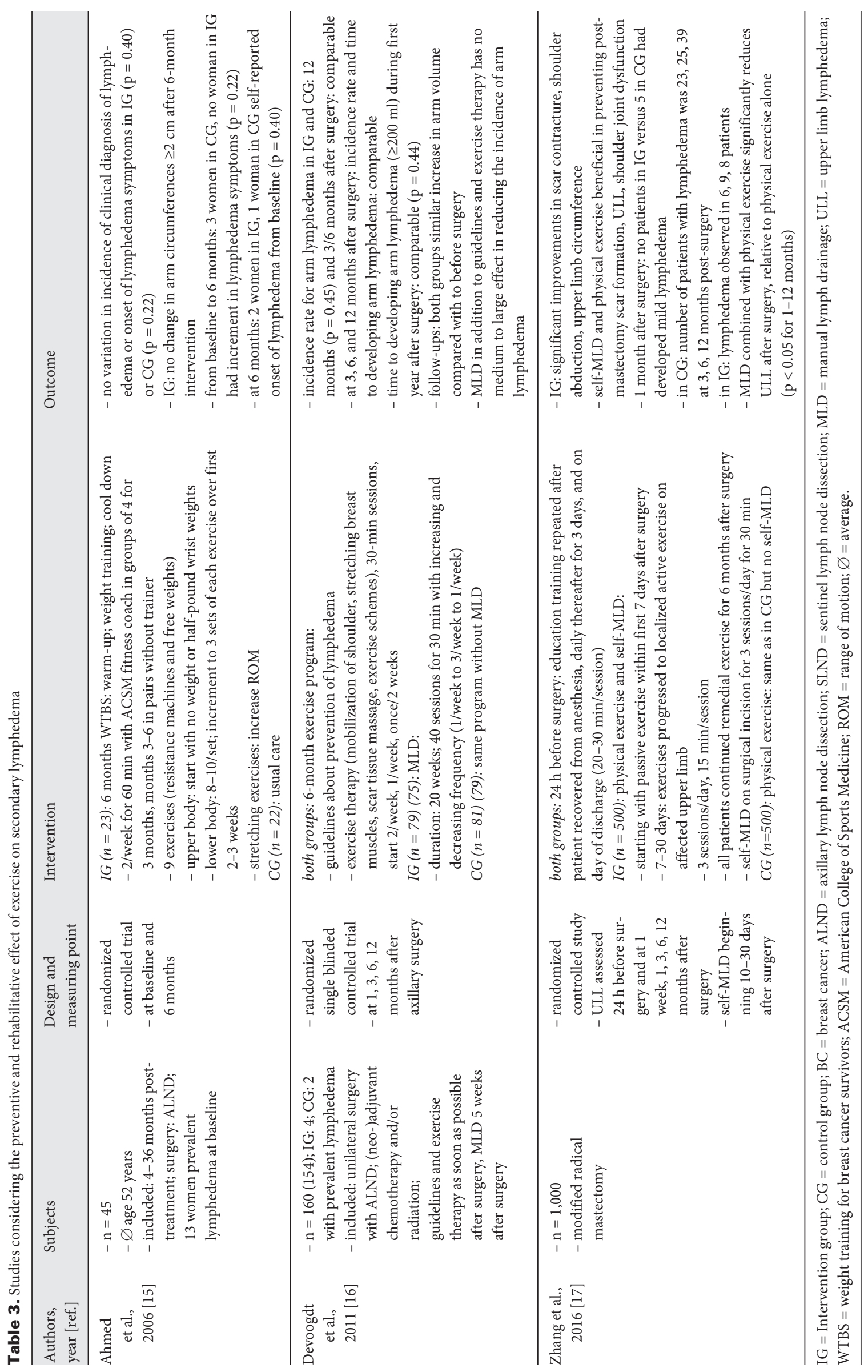




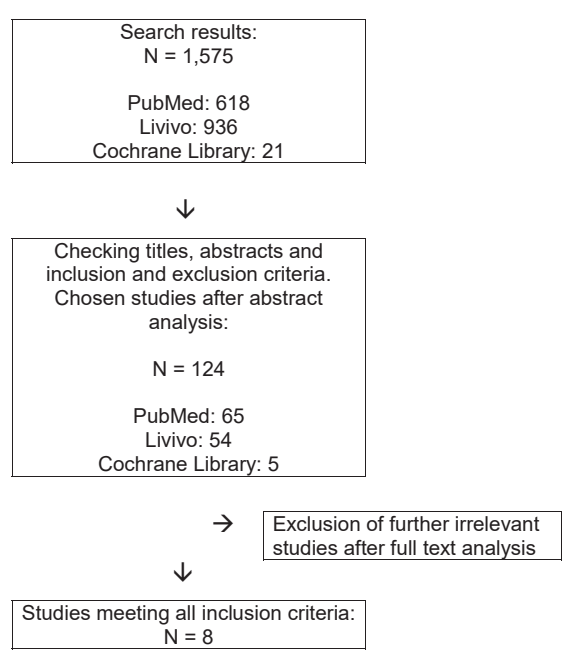

studies for systematic review.

(MLD), stretching, massage, and/or kinesiotherapy $[5,13,14,16$, 17]. Resistance training started between 4 months and 5 years after the end of primary treatment. 2 studies started combined exercise therapy $24-48 \mathrm{~h}$ after surgery. Intervention programs lasted from a minimum of 3 weeks to a maximum of 12 months. MLD in addition to exercise was administered in 4 studies $[5,14,16,17]$.

Preventive effects of exercise in relation to SL in breast cancer were observed. Significant effects could be identified in 5 studies $[5,11,13,14,17] .3$ studies recognized significant effects of exercise in combination with MLD [5, 14, 17]. Schmitz et al. [11] reported only a minor incidence of SL in the intervention group undergoing a 1-year (twice weekly) progressive weightlifting intervention compared to the control group. This difference was even bigger in women who had at least 5 lymph nodes removed. The study conducted by Torres Lacomba et al. [5] observed a significantly lower incidence of lymphedema in the intervention group receiving physiotherapy (MLD, massage, stretching, progressive exercise, and educational therapy) compared to the control group receiving only educational material. Moreover, in the control group, onset of lymphedema was diagnosed 4 times earlier compared to the exercise group [5]. In the study by De Rezende et al. [13], the intervention group showed statistically significant better recovery of flexion, abduction, and external movements in the shoulder compared to the control group. Zimmermann et al. [14] performed a 6-month MLD intervention and showed 6 months after surgery a significant increase in arm volume on the operated side in the control group. Zhang et al. [17] combined physical exercise with self-administered MLD in their trial and showed that the combined intervention significantly reduced upper limb lymphedema after surgery compared to physical exercise alone.

\section{Discussion}

The aim of this study was to analyze the potential preventive effects of exercise-based therapies on SL incidence after breast cancer. To our knowledge, a systematic review on solely the topic of prevention was not conducted before. 8 randomized controlled studies [5, 11-17] could be identified. They showed that exercise in the form of progressive resistance training as well as combined exercise therapies consisting of physiotherapy, physical therapy, MLD, stretching, massage, and/or kinesiotherapy are safe and might have a preventive effect on SL incidence.

In the past, physicians believed that cancer patients must avoid exercise [8], and literature indicating that exercise can cause or exacerbate lymphedema still exists [15]. However, the present review revealed that 5 out of 8 trials reported significant preventive effects of resistance training and exercise on SL incidence. Park et al. [18] investigated the incidence and risk factors of SL in breast cancer patients. They demonstrated that women who exercised regularly, performed preventive self-care, and received information about the possible appearance of a lymphedema before local treatment had a lower risk of developing lymphedema. Proposed mechanisms included that exercise promotes the contractility of the skeletal muscles and subsequently provides primary pump mechanisms for lymph and venous drainage $[19,20]$.

Besides radiation and the number of surgically removed lymph nodes [16], overweight also contributes crucially to the development of SL [21-23]. In the study by Sagen et al. [12], a significant risk increase was observed in patients with a body mass index of $>25 \mathrm{~kg} / \mathrm{m}^{2}(\mathrm{p}=0.005)$. Shaw et al. [24] also referred to the relationship between overweight/obesity and the development of lymphedema [25]. According to Bicego et al. [19], further risk factors include obstruction, trauma, and inflammation [26]. Physical inactivity results in a decrease in lymph circulation. Physical exercise maintaining or improving the 'range of motion' of the shoulder therefore seems to be an effective and preventive measure. Additional benefits include improved muscle strength/fitness and maintenance of body weight.

A limitation of this systematic review is that probably not all studies covering the preventive effect of exercise on SL in breast cancer were identified in the literature. Also, we must take into consideration that other risk factors contribute to the development of SL as previously described. In this review, we included studies performed between 2006 and 2016 during which period surgical treatment and especially axillary staging shifted from axillary dissection level I-III to sentinel lymph node dissection. The risk of SL decreased during that time, making it difficult to compare these studies.

Due to the fact that the included studies evaluated different exercise intervention regimens, we are currently unable to provide clear and evidence-based exercise recommendations. The results published by Cavanaugh [27] underline the urgency of individualizing exercise guidelines. Further, Ahmed et al. [15] recommend that breast cancer patients should perform strength training of the upper body because this does not promote the risk or symptoms of lymphedema. Besides, Sagen et al. [12] recommend that patients with axillary lymph node dissection continue to exercise without restriction in daily living. In addition, considering early exercise intervention in women with breast cancer is important and necessary [27]. The studies by Ahmed et al. [15] and Schmitz et al. [11] show that progressive strength training can generate a preventive 
effect. Combined exercise therapy can result in similar effects [5, 14, 17]. Exercise additionally supports muscular pump function and should be performed at a moderate level of intensity and with a small number of repetitions. The application of MLD seems to have prophylactic effects only in combination with exercise, and current data does not show any evidence for MLD as a single primary prophylactic method [28]. To guarantee the safe and effective performance of the exercises, the support of a certified exercise therapist during the first months of the strength training is also advised [29].

\section{Conclusion}

A total of 8 randomized controlled trials yielded promising data supporting the preventive effect of exercise on SL in breast cancer patients. Results showed that exercise is safe and that it is important to maintain exercise in daily living. Exercise in the form of progressive resistance training as well as combined exercise therapies are safe and might have a preventive effect on SL incidence. Future investigations will have to differentiate between patients with and without axillary surgery, and exact exercise recommendations for therapists and the rehabilitation system should be based on further studies.

\section{Disclosure Statement}

The authors declared no potential conflicts of interest with respect to the research, authorship, and/or publication of this article.

\section{References}

1 Fitzmaurice C, Dicker D, Pain A; Global Burden of Disease Cancer Collaboration: The Global Burden of Cancer 2013. JAMA Oncol 2015;1:505-227.

2 Nelson NL: Breast cancer-related lymphedema and resistance exercise: a systematic review. J Strength Cond Res 2016;30:2656-2665.

3 Bennett Britton TM, Purusgitham AD: Understanding breast cancer-related lymphoedema. Surgeon 2009; $120-124$.

4 Schmitz KH: Balancing lymphedema risk: exercise versus deconditioning breast cancer survivors. Exerc Sport Sci Rev 2010;38:17-24

5 Torres Lacomba M, Yuste Sánchez MJ, Zapico Goñi Á Prieto Merino D, Mayoral del Moral O, Cerezo Téllez E, Minayo Mogollón E: Effectiveness of early physiotherapy to prevent lymphoedema after surgery for breast cancer: randomised, single blinded, clinical trial. BMJ 2010;340:b5396.

6 Mortimer PS: The pathophysiology of lymphedema. Cancer 1998;83:2798-2802.

7 Francis WP, Abghari P, Du W, Rymal C, Suna M, Kosir MA: Improving surgical outcomes: standardizing the reporting of Incidence severity of acute lymphedema after sentinel lymph node biopsy and axillary lymph node dissection. Am J Surg 2006;192: 636-639.

8 Schmitz KH, Courneya KS, Matthews C, DemarkWahnefried W, Galvao DA, Pinto BM, Irwin ML, Wolin KY, Segal RJ, Lucia A, Schneider CM, von Gruenigen VE, Schwartz AL; American College of Sports Medicine: American College of Sports Medicine roundtable on exercise guidelines for cancer survivors. Med Sci Sports Exerc. 2010;42:1409-1426.

9 Keilani M, Hasenoehrl T, Neubauer M, Crevenna R: Resistance exercise and secondary lymphedema in breast cancer survivors - a systematic review. Support Care Cancer 2016;24:1907-1916.

10 Schmitz KH, Ahmed RL, Troxel A, Cheville A, Smith R, Lewis-Grant L, Bryan CJ, Williams-Smith CT, Greene QP: Weight lifting in women with breast-cancer-related lymphedema. N Engl J Med 2009;361:664673.
11 Schmitz KH, Ahmed RL, Troxel AB, Cheville A, LewisGrant L, Smith R, Bryan CJ, Williams-Smith T, Chittams J: Weight lifting for women at risk for breast cancer-related lymphedema. JAMA 2010;304:2699-2705.

12 Sagen A, Karesen R, Risberg MA: Physical activity for the affected limb and arm lymphedema after breast cancer surgery. A prospective, randomized controlled trial with two years follow-up. Acta Oncol 2009;48: 1102-1110.

13 De Rezende LF, Franco RL, de Rezende MF, Beletti PO, Morais SS, Gurgel MS: Two exercise schemes in postoperative breast cancer: comparison of effects on shoulder movement and lymphatic disturbance. Tumori 2006;92:55-61.

14 Zimmermann A, Wozniewski M, Szklarska A, Lipoxicz A, Szuba A: Efficacy of manual lymphatic drainage in preventing secondary lymphedema after breast cancer surgery. Lymphology 2012;45:103-112.

15 Ahmed RL, Thomas W, Yee D, Schmitz KH: Randomized controlled trial of weight training and lymphedema in breast cancer survivors. J Clin Oncol 2006;24:2765-2772.

16 Devoogdt N, Christiaens MR, Geraerts I, Truijen S, Smeets A, Leunen K, Neven P, Van Kampen M: Effect of manual lymph drainage in addition to guidelines and exercise therapy on arm lymphoedema related to breast cancer: randomised controlled trial. BMJ 2011; 343:d5326.

17 Zhang L, Fan A, Yan J, He Y, Zhang H, Zhang H, Zhong Q, Liu F, Luo Q, Zhang L, Tang H, Xin M: Combining manual lymph drainage with physical exercise after modified radical mastectomy effectively prevents upper limb lymphedema. Lymphat Res Biol 2016;4:104-108.

18 Park JH, Lee WH, Chung HS: Incidence and risk factors of breast cancer lymphoedema. J Clin Nurs 2008; 17:1450-1459.

19 Bicego D, Brown C, Ruddick M, Storey D, Wong C, Harris SR: Exercise for women with or at risk for breast cancer-related lymphedema. Phys Ther 2006;86: 1398-1405.
20 Witte CL, Witte MH: Contrasting patterns of lymphatic and blood circulatory disorders. Lymphology 1987;20:171-178.

21 Erickson VS, Pearson ML, Ganz PA, Adams J, Kahn KL: Arm edema in breast cancer patients. J Natl Cancer Inst 2001;93:96-111.

22 Hayes SB, Freedman GM, Li T, Anderson PR, Ross E: Does axillary boost increase lymphedema compared with supraclavicular radiation alone after breast conservation? Int J Radiat Oncol Biol Phys 2008;72:14491455.

23 Paskett ED, Naughton MJ, McCoy TP, Case LD, Abbott JM: The epidemiology of arm and hand swelling in premenopausal breast cancer survivors. Cancer Epidemiol Biomarkers Prev 2007;16:775-782.

24 Shaw C, Mortimer P, Judd PA: A randomized controlled trail of weight reduction as a treatment for breast cancer-related lymphedema. Cancer 2007;110: 1868-1874.

25 Ochalek K: Prevention of lymphoedema. Wspolczesna Onkol 2011;15:354-356.

26 Hull MM: Lymphedema in women treated for breast cancer. Semin Oncol Nurs 2000;16:226-237.

27 Cavanaugh KM: Effects of early exercise on the development of lymphedema in patients with breast cancer treated with axillary lymph node dissection. J Oncol Pract 2011;7:89-93.

28 Stuiver MM, ten Tusscher MR, Agasi-Idenburg CS, Lucas C, Aaronson NK, Bossuyt PM: Conservative interventions for preventing clinically detectable upperlimb lymphoedema in patients who are at risk of developing lymphoedema after breast cancer therapy. Cochrane Database Syst Rev 2015;CD009765.

29 Schmitz KH, Ahmed RL, Hannan PJ, Yee D: Safety and efficacy of weight training in recent breast cancer survivors to alter body composition, insulin, and insulinlike growth factor axis proteins. Cancer Epidemiol Biomarkers Prev 2005;14:1672-1680. 